\title{
Influence of Circadian Rhythm on Thrombus Formation of Murine Deep Vein Thrombosis Model
}

\author{
Nosaka $\mathbf{M}^{*}$, Ishida Y, Kimura A, Yamamoto H, \\ Kuninaka Y, Shimada E, Okumoto K, Okada M, \\ Hata S, and Kondo T \\ Department of Forensic Medicine, Wakayama Medical \\ University, Japan \\ *Corresponding author: Nosaka M, Department \\ of Forensic Medicine, Wakayama Medical University, \\ Wakayama, Japan
}

Received: August 22, 2017; Accepted: September 09, 2017; Published: October 12, 2017

\begin{abstract}
Deep vein thrombosis and pulmonary embolism are major health problems associated with high mortality. In previous studies, how the time of therapy or drug delivery can impact treatment effect and outcomes. This study was to determine whether stasis-started time influenced thrombus formation and resolution response. The mice ligated at 12:00 PM had decreased survival rate than the mice ligated at 7:00 AM. The thrombus size of at 12:00 PM-ligated mice were larger than thrombi of at 7:00-AM-ligated ones. In 12:00 PM-ligated mice, the Mmp9 gene expressions were lower than 7:00 AM-ligated ones. The treatment time may affect response in mice receiving stasis for formation of thrombi. Subsequent chronotherapy studies in anticoagulant therapy should investigate the time of therapy or medication.
\end{abstract}

Keywords: Deep vein thrombosis; Pulmonary thromboembolism; Intravenous thrombus

\section{Introduction}

Deep vein thrombus (DVT) is a major problem as the cause of pulmonary thromboembolism (PTE) from both clinical and forensic aspects, and it is multifactorial and often results from a combination of risk factors such as genetic conditions, obesity, drugs, pregnancy, aging, trauma, and malignancy. DVT is frequently complicated with severe morbidity, leading sometimes to mortality. But the start time of the event that was a critical turning point and severity of thrombosis was not clear. Recently, in the researches were performed on circadian rhythm or chronotherapy. In this study, we found out the influence of the time of surgical operation and stasis-state for thrombus formation using stasis-induced murine DVT model.

\section{Methods}

\section{Mice}

Pathogen-free 8- to 10 -week-old male $\mathrm{BALB} / \mathrm{c}$ mice were obtained from SLC and designated as WT mice in this study. All mice were bred and housed at a constant temperature $\left(23 \pm 2{ }^{\circ} \mathrm{C}\right)$, with a 12-h light/dark cycle (light on at 8:00 AM and off at 8:00 PM). They were fed with standard feed and given water ad libitum. All animal experiments were approved by the Committee on Animal Care and Use at Wakayama Medical University.

\section{Stasis-induced DVT model}

Intravenous thrombus was induced as described previously. Briefly, after deep anesthesia with intraperitoneal injection of 2,2,2-tribromoethanol (Avertin, $240 \mathrm{mg} / \mathrm{kg}$ body weight), a $1-\mathrm{cm}$ incision was made along the abdominal midline, and the inferior vena cava (IVC) was exposed and ligated with 3-0 silk suture. The abdominal wall was closed, and $1 \mathrm{ml}$ of PBS was injected subcutaneously [1-3]. The ligation time was at 7:00 AM and at 12:00 $\mathrm{PM}$. This operation was performed within 7 minutes to exclude an effect of the ligation time lag.

\section{Immunohistochemical staining}

Intravenous thrombi, obtained at the indicated time intervals after IVC ligation, were fixed in $4 \%$ formaldehyde buffered with PBS (pH 7.2) and transversely cut in the middle of the thrombus, and paraffinembedded sections (4- $\mu \mathrm{m}$ thick) were made. Immunostaining was automatically performed by Ventana Discovery XT (Ventana Medical Systems, Inc., AZ). The primary Abs were diluted with the blocking buffer (PBS containing 1\% normal serum corresponding to the secondary Abs and $1 \%$ bovine serum albumin) to reduce nonspecific reactions. Thereafter, the sections were reacted with antiMPO mAbs at $37^{\circ} \mathrm{C}$ for $60 \mathrm{~min}$. After incubation with biotinylated secondary Absat $37{ }^{\circ} \mathrm{C}$ for $60 \mathrm{~min}$, the DAB Map kit (Ventana Medical Systems, Inc., AZ) was used to visualize the antigens for all stains. Subsequently, all slides were counterstained with hematoxylin or lithium carbonate to visualize the nuclei [3-5].

\section{Extraction of total RNAs and real-time RT-PCR}

Real-time RT-PCR was performed as described previously. Briefly, total RNA was extracted from thrombi samples using ISOGEN (Nippon Gene) according to the manufacturer's instructions, and $1 \mu \mathrm{g}$ of total RNA was reverse transcribed into cDNA. Thereafter, generated cDNA was subjected to real-time PCR analysis using SYBR Premix Ex Taq II kit (Takara Bio) with the specific primers sets. Relative quantity of target gene expression to $\beta$-actin gene was measured by comparative Ct method [6].

\section{Measurement of PT and APTT}

At 7:00 AM or at 12:00 PM, the blood was obtained from the hearts of mice. These blood samples were taken with $3.8 \%$ citrate solution and centrifuged to obtain plasma samples. PT and APTT of citrated plasma samples were measured by using COAGSEARCH (A\&T) according to the manufacturer's instructions [6].

\section{Results}

We found out the mice reacted slowly to anesthesia at 7:00 AM, 


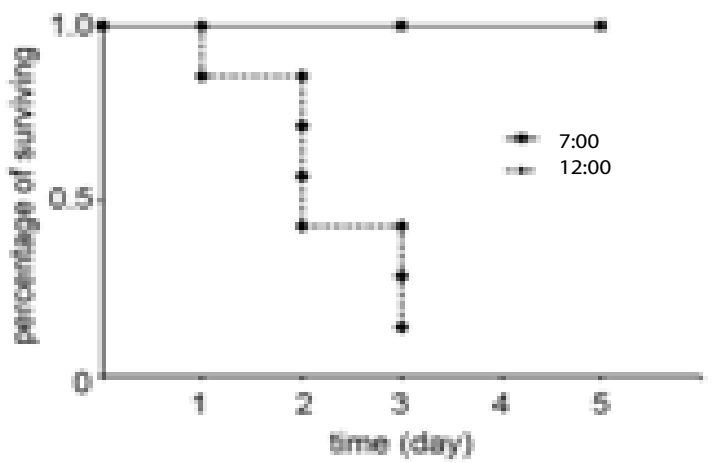

Figure 1: Survival rate of Kaplan Meier method. There were significan difference $(P<0.01)$ between 7:00 AM-ligated mice and 12:00 PM-ligate ones.

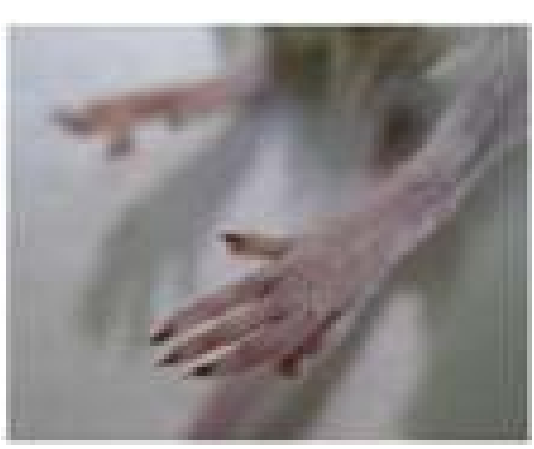

Figure 2: Subungual hematoma of a 12:00 PM-ligated mouse.
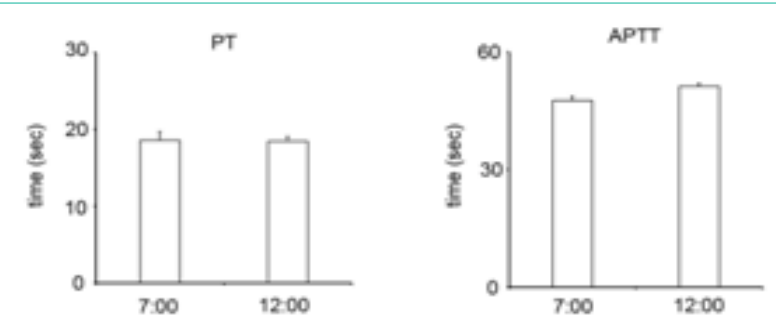

Figure 3: PT and APTT. There were no significant differences between at 7:00 AM and 12:00 PM in mice.
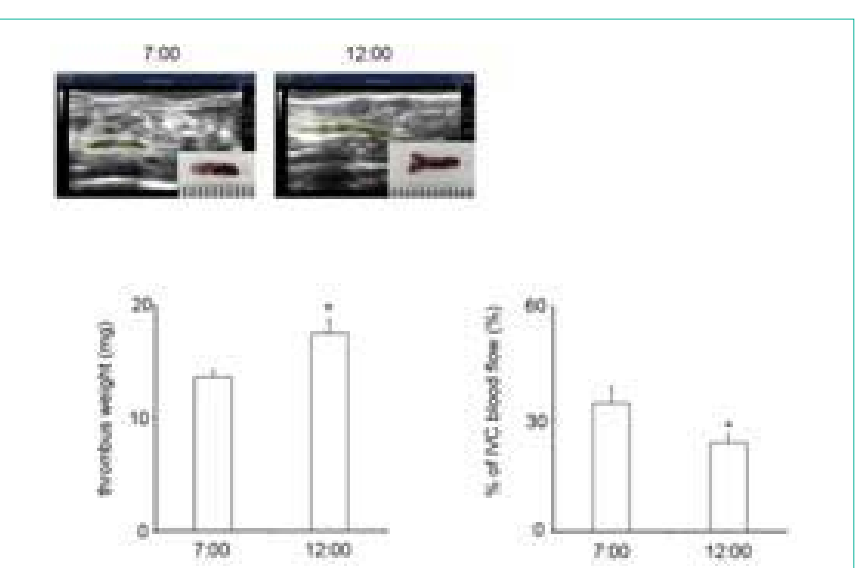

Figure 4: Thrombus size (mg) and IVC blood flow (\%). All values represent mean \pm SEM $(n=5)$. There were significant difference in thrombus size and IVC blood flow between 7:00 AM-ligated mice and 12:00 PM-ligated ones. ${ }^{*} \mathrm{P}<0.05$.

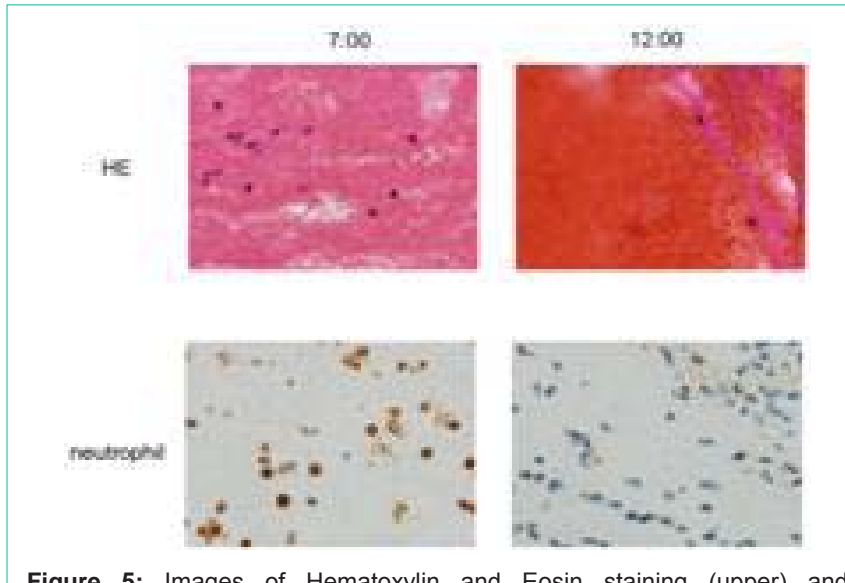

Figure 5: Images of Hematoxylin and Eosin staining (upper) and immunohistochemical staining for neutrophil (lower). Their normal number of neutrophils in the thrombi of 7:00 AM-ligated mice. (Original magnification; x400).

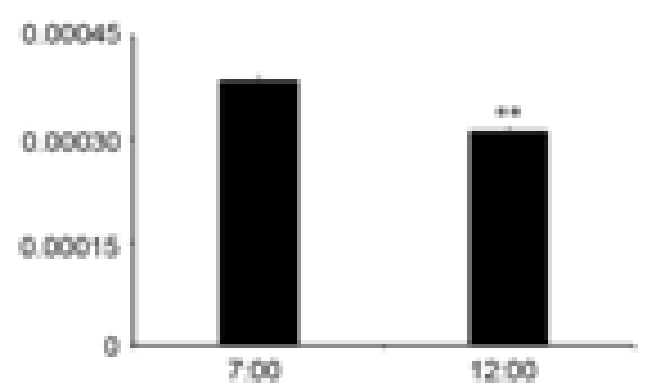

Figure 6: Intrathrombotic Mmp9 gene expressions by real-time RT-PCR as described in Methods. All values represent mean \pm SEM $(n=5)$. There were significant difference between 7:00 AM-ligated mice and 12:00 PM-ligated ones. ${ }^{*} \mathrm{P}<0.01$.

but reacted normally at 12:00 PM, without a bad influence for the operation on both time. The mice ligated at 12:00 PM had decreased survival rate than the mice ligated at 7:00 AM (Figure 1). And at 12:00 PM ligated dead mice had subungual hemorrhage and lung hemorrhage (Figure 2). But there were no significant differences on PT and APTT, between 7:00 AM-ligated mice and at 12:00 PM -ligated ones (Figure 3). The thrombus size of at noon-ligated mice was larger than thrombi of at 7:00-AM-ligated ones (Figure 4).

We detected the histopathological and immunohistochemical changes, at 12:00 PM -ligated thrombi had poor neutrophils than 7:00 AM-ligated ones on H-E staining images and immunohistochemical staining images for MPO (Figure 5). At 5-day, intrathrombotic Mmp9 gene expressions were detected irrespectively the time of ligations. In 12:00 PM-ligated mice, the Mmp9 gene expressions were lower than 7:00 AM-ligated ones (Figure 6).

\section{Discussion}

The circadian rhythm is generated by a central circadian oscillator in the suprachiasmatic nucleus of the hypo-thalamus. The circadian rhythm in biological, physiological, and behavioral processes has been found in organisms of animalia, plantae, fungi and algae [7]. We faced an age of the genome-based drug discovery, and develop of the molecular target drug. These trends show possibility of the chronotherapy forpeptic ulcer, asthma bronchiale, angina inversa, 
migraine, allergic rhinitis, rheumatic gout, depressive illness, cardiac infarct, brain infarct, osteoarthropathy and cutaneous hypersensitivity $[8,9]$.

We found out the thrombus size, blood flow and $M m p 9$ gene expression of deep vein thrombi using murine model with different time of stasis-started. At 12:00 PM, the operation and stasis were more invasive than 7:00 AM. There was more number of neutrophils in the thrombi of 7:00 AM-ligated mice than 12:00 PM-ligated ones. Intrathrombotic neutrophils are recruited to developing thrombi In recent years, neutrophil extracellular traps (NETs) have been identified as major triggers and structural factors of thrombi $[5,10,11]$. They amplify intravascular coagulation by stimulating the tissue factor-dependent extrinsic pathway via inactivation of endogenous anticoagulants, enhancing factor XII activation or decreasing plasmin generation. And intrathrombotic neutrophils were important for preventing infection. 7:00 AM-ligated mice would be protected from infection by intrathrombotic neutrophils. Because these present results were obtained from well-controlled nocturnality animal experiments, it is of course necessary to perform further study using human thrombus samples. In various disease models, we should confirm the disease-induced time and the association of the survival rate and invasion level.

\section{References}

1. Nosaka M, Ishida $Y$, Kimura $A$, Kawaguchi $T$, Yamamoto $H$, Kuninaka $Y$ et al. Immunohistochemical detection of intrathrombotic fibrocytes and its application to thrombus age estimation in murine deep vein thrombosis model. Int J Legal Med. 2016; 131: 179-183.
2. Nosaka M, Ishida Y, Kimura A, Hama M, Kawaguchi T, Yamamoto H, Kuninaka Y, Shimada E, et al. Immunohistochemical detection of intrathrombotic IL-6 and its application to thrombus age estimation. Int J Legal Med. 2015; 129 . 1021-1025

3. Nosaka M, Ishida Y, Kimura A, Kondo T. Immunohistochemical detection of intrathrombotic macrophage-derived cytokines and its application to thrombus age estimation in murine deep vein thrombosis model. Int $\mathrm{J}$ Legal Med. 2013; 127: 937-942

4. Nosaka M, Ishida $\mathrm{Y}$, Kimura A, Kondo T. Time-dependent appearance of intrathrombus neutrophils and macrophages in a stasis-induced deep vein thrombosis model and its application to thrombus age determination. Int $\mathrm{J}$ Legal Med. 2009; 123: 235-240.

5. Nosaka M, Ishida Y, Kimura A, Kuninaka Y, Inui M, Mukaida N, et al. Absence of IFN-gamma accelerates thrombus resolution through enhanced MMP-9 and VEGF expression in mice. J Clin Invest. 2011; 121: 2911-2920.

6. Kimura A, Ishida Y, Hayashi T, Nosaka M, Kondo T. Estimating time of death based on the biological clock. Int J Legal Med. 2011; 125: 385-391.

7. Heran BS, Allan GM, Green L, Korownyk C, Kolber M, Olivier N, et al. Effect of medication timing on anticoagulation stability in users of warfarin (the INRange RCT): study protocol for a randomized controlled trial. 2016; 17 : 391.

8. Ando H, Otoda T, Ookami H, Nagai Y, Inano A, Takamura T, et al. Dosing time-dependent effect of raloxifene on plasma plasminogen activator inhibitor-1 concentrations in post-menopausal women with osteoporosis. Clin Exp Pharmacol Physiol. 2013; 40: 227-232.

9. Martinod K, Demers M, Fuchs TA, Wong SL, Brill A, Gallant M, et al. Neutrophi histone modification by peptidylarginine deiminase 4 is critical for deep vein thrombosis in mice. Proc Natl Acad Sci U S A. 2013; 110: 8674-8679.

10. Fuchs TA, Brill A, Wagner DD. Neutrophil extracellular trap (NET) impact on deep vein thrombosis. Arterioscler Thromb Vasc Biol. 2012; 32: 1777-1783.
Ann Hematol Oncol - Volume 4 Issue 9 - 2017

ISSN : 2375-7965 | www.austinpublishinggroup.com

Nosaka et al. (C) All rights are reserved
Citation: Nosaka M, Ishida Y, Kimura A, Yamamoto H, Kuninaka Y, Shimada E, et al. Influence of Circadian Rhythm on Thrombus Formation of Murine Deep Vein Thrombosis Model. Ann Hematol Oncol. 2017; 4(9): 1171. 